\title{
Correction to: Endoscopic Argon Plasma Coagulation Vs. Multidisciplinary Evaluation in the Management of Weight Regain after Gastric Bypass Surgery: a Randomized Controlled Trial with SHAM Group
}

\author{
Luiz Gustavo de Quadros ${ }^{1,2,3,4}$. Manoel Galvão Neto ${ }^{1,5}$ • João Caetano Marchesini ${ }^{1}$ • André Teixeira ${ }^{6}$. \\ Eduardo Grecco ${ }^{1}$ • Roberto Luiz Kaiser Junior ${ }^{7,8}$ - Natan Zundel ${ }^{9}$ - Idiberto José Zotarelli Filho ${ }^{8}$. \\ Thiago Ferreira de Souza ${ }^{10}$. Admar Concon Filho ${ }^{1}$. Lyz Bezerra da Silva ${ }^{4}$ - Almino Cardoso Ramos ${ }^{1,11}$. \\ Álvaro Antônio Bandeira Ferraz ${ }^{4}$. Josemberg Marins Campos ${ }^{4}$
}

Published online: 19 May 2020

C) Springer Science+Business Media, LLC, part of Springer Nature 2020

\section{Correction to: Obesity Surgery.}

https://doi.org/10.1007/s11695-020-04414-6

In the original article the captions for Figs. 1-3 are incorrect. The correct captions are as follows:

The online version of the original article can be found at https://oi.org/ 10.1007/s11695-020-04414-6

Luiz Gustavo de Quadros

Gustavo_quadros@hotmail.com

Manoel Galvão Neto

galvaon@gmail.com

João Caetano Marchesini

jcmarchesini@gmail.com

André Teixeira

ateixeira200@yahoo.com

Eduardo Grecco

dreduardogrecco@gmail.com

Roberto Luiz Kaiser Junior

kaiserjr@kaiserclinica.com.br

Natan Zundel

drnazuma99@yahoo.com

Idiberto José Zotarelli Filho

m.zotarelli@gmail.com

Thiago Ferreira de Souza

tsouzahc@gmail.com

Admar Concon Filho

conconaf@gmail.com

Lyz Bezerra da Silva

lyzbezerra@gmail.com
Almino Cardoso Ramos

ramos.almino@gmail.com

Álvaro Antônio Bandeira Ferraz

alvaroabferraz@gmail.com

Josemberg Marins Campos

josembergcampos@gmail.com

Faculty of Medicine of ABC, Santo Andre, São Paulo, Brazil

2 Beneficência Portuguesa Hospital, Sao Jose do Rio Preto, São Paulo, Brazil

3 Kaiser Day Hospital, Sao Jose do Rio Preto, São Paulo, Brazil

4 Federal University of Pernambuco, Recife, Pernambuco, Brazil

5 Endovitta Institute, Sao Paulo, São Paulo, Brazil

6 Orlando Health Hospital, Orlando, USA

7 Beneficência Portuguesa Hospital, Sao Jose do Rio Preto, São Paulo, Brazil

8 Kaiser Day Hospital, Sao Jose do Rio Preto, São Paulo, Brazil

9 Jackson North Medical Center, University at Buffalo, Miami, USA

10 Hospital das Clínicas da Faculdade de Medicina, Universidade de São Paulo, Sao Paulo, Brazil

11 Gastro Obeso Center, Sao Paulo, Brazil 
Assessed for Eligibility $(n=144)$

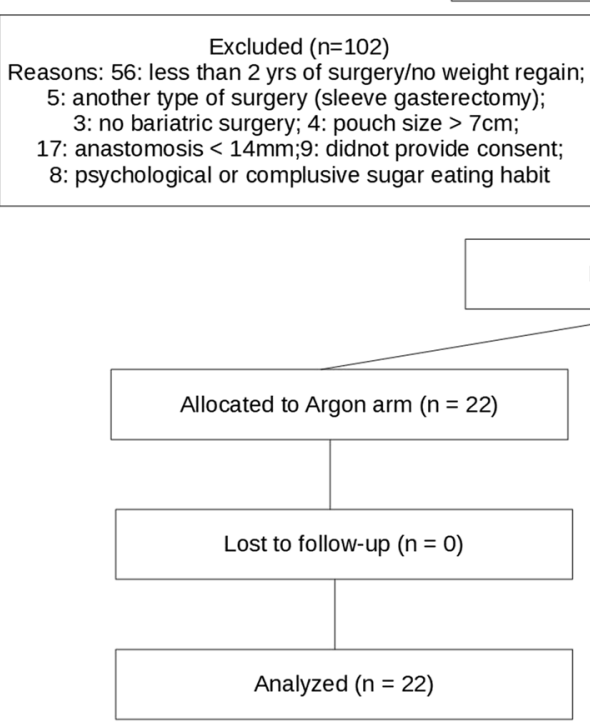

Randomized ( $n=42)$

Fig. 1 Study flowchart

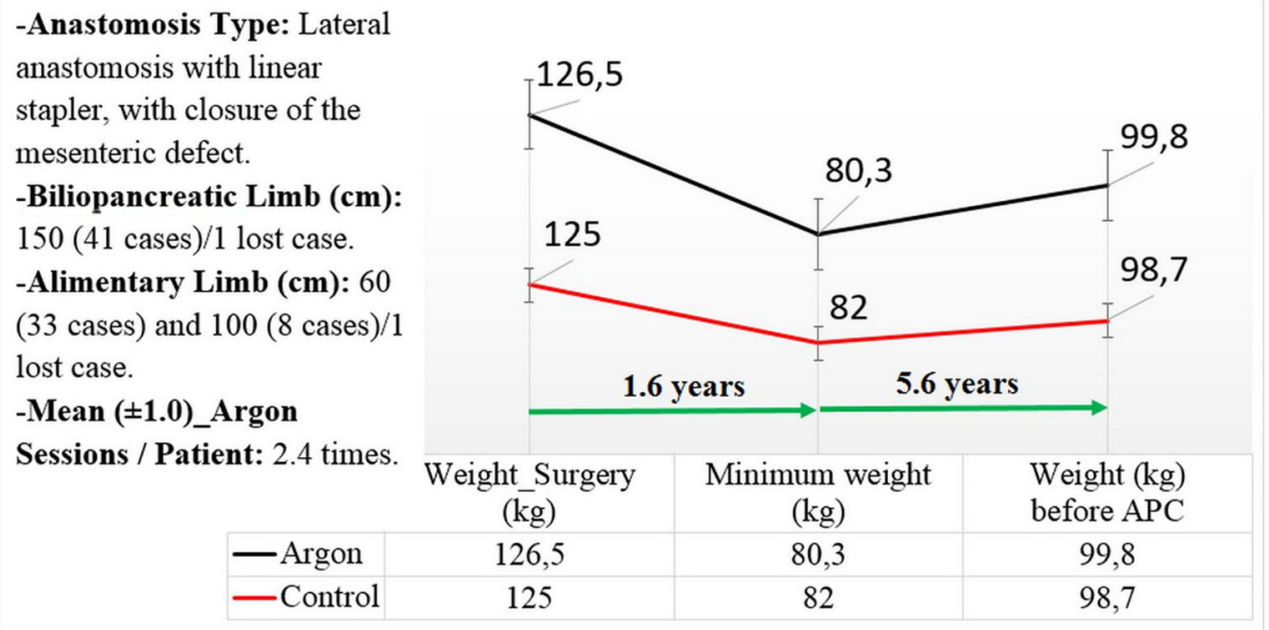

Fig. 2 Bariatric surgery characteristics and APC sessions 


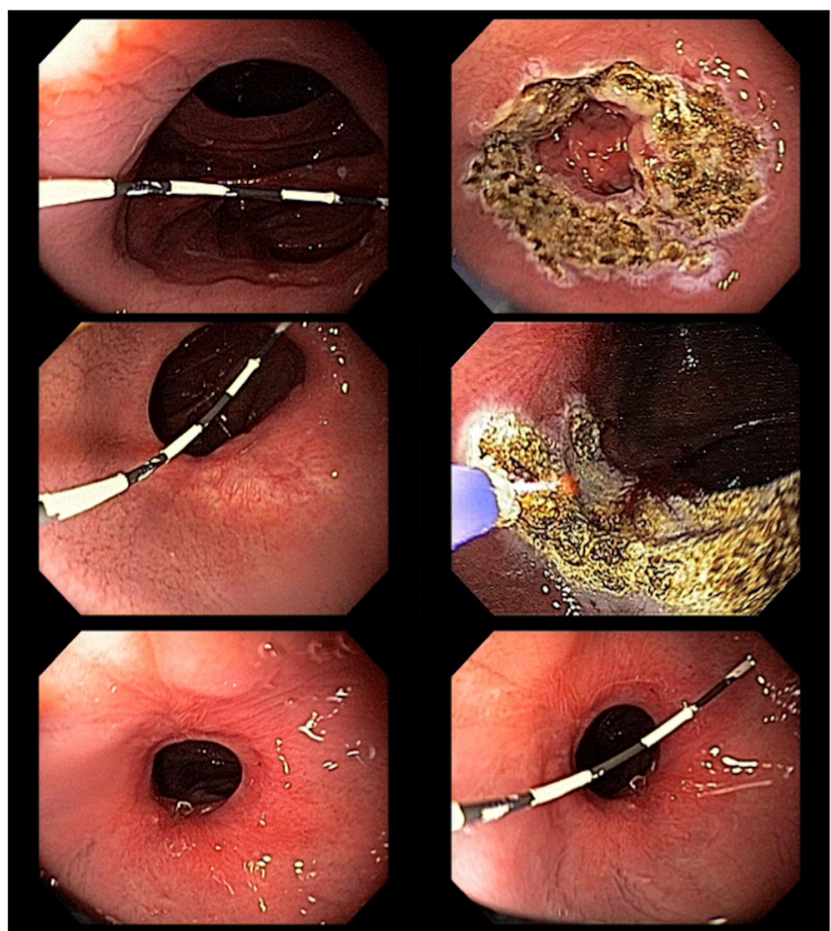

Fig. 3 APC sessions using a validated ruler for measurement, showing anastomosis narrowing after 2 argon sessions

Publisher's Note Springer Nature remains neutral with regard to jurisdictional claims in published maps and institutional affiliations. 